\title{
Planet formation around M-dwarfs: the moving snow line and super-Earths
}

\author{
Grant M. Kennedy $\cdot$ Scott J. Kenyon • Benjamin \\ C. Bromley
}

Received: 16 March 2007 / Accepted: 26 June 2007 / Published online: 2 August 2007

(C) Springer Science+Business Media B.V. 2007

\begin{abstract}
Planets result from a series of processes within a circumstellar disk. Evidence comes from the near planar orbits in the Solar System and other planetary systems, observations of newly formed disks around young stars, and debris disks around main-sequence stars. As planethunting techniques improve, we approach the ability to detect systems like the Solar System, and place ourselves in context with planetary systems in general. Along the way, new classes of planets with unexpected characteristics are discovered. One of the most recent classes contains super Earth-mass planets orbiting a few AU from low-mass stars. In this contribution, we outline a semi-analytic model for planet formation during the pre-main sequence contraction phase of a low-mass star. As the star contracts, the "snow line", which separates regions of rocky planet formation from regions of icy planet formation, moves inward. This process enables rapid formation of icy protoplanets that collide and merge into super-Earths before the star reaches the main sequence. The masses and orbits of these superEarths are consistent with super-Earths detected in recent microlensing experiments.
\end{abstract}

G.M. Kennedy ( $\varangle)$

Research School of Astronomy and Astrophysics, Mt Stromlo Observatory, Australian National University, Cotter Road, Weston Creek, ACT 2611, Australia

e-mail: grant@mso.anu.edu.au

\section{S.J. Kenyon}

Smithsonian Astrophysical Observatory, Cambridge, MA 02138, USA

e-mail: kenyon@cfa.harvard.edu

B.C. Bromley

Department of Physics, University of Utah, 201 JFB,

Salt Lake City, UT 84112, USA

e-mail: bromley@physics.utah.edu
Keywords Planetary systems: formation · Planetary systems: protoplanetary disks $\cdot$ Stars: evolution $\cdot$ Stars: formation

\section{Introduction}

Planets form in circumstellar disks. The strongest evidence is contained in the nearly flat orbital structure of the Solar System. The near ubiquity of infra-red excesses around young stars (Haisch et al. 2000)—starlight reprocessed by a dusty disk-argues strongly for planetary formation as a common and robust process.

The abundance of low-mass stars in the Milky Way, and evidence that they harbour circumstellar disks and planets, makes them potentially fruitful and important locales for planet formation. The recent microlensing discoveries of $\sim 5-10 M_{\oplus}$ planets orbiting at distances of a few AU provide additional evidence that planetary systems with planet/star mass-ratios similar to Neptune/Sun are common (Beaulieu et al. 2006; Gould et al. 2006).

The discovery of super Earth-mass planets-dubbed "super-Earths"-around low-mass stars challenges our understanding of planet formation. With orbital semi-major axes $a \sim 2-3 \mathrm{AU}$, these planets are probably ice giants roughly similar in structure to Uranus and Neptune in the Solar System.

Boss (2006) proposes that these planets form in two stages. After gravitational instability produces a gas giant, photoevaporation of the gas giant atmosphere leaves behind an icy core with $M \sim 10-20 M_{\oplus}$. This mechanism requires a massive disk to initiate the instability and a nearby O-type star to photoevaporate the giant planets atmosphere. Boss notes that this process should yield (i) super-Earths around 
$\mathrm{M}$ dwarfs formed in rich star clusters and (ii) gas giants around $\mathrm{M}$ dwarfs formed in low-mass stellar associations.

Beaulieu et al. suggest that super-Earths favor coagulation models, where collisions of $1-10 \mathrm{~km}$ objects eventually produce icy planets with $M \sim 10 M_{\oplus}$ at 1-10 AU. Although numerical calculations appear to preclude gas giants at $1-10$ AU around M dwarfs (Laughlin et al. 2004), there has been no demonstration that coagulation produces icy planets on reasonable timescales in a disk around an $\mathrm{M}$ dwarf.

Here we outline a semi-analytic coagulation model, which shows that contraction of the central star along a premain sequence (PMS) Hayashi track sets the initial conditions for planet formation around low-mass stars. Our results indicate that icy protoplanets with $M \sim 0.1-1 M_{\oplus}$ form in $\sim 0.1-1$ Myr at 1-4 AU. Over 50-500 Myr, collisions between protoplanets produce super-Earths with masses similar to those detected in microlensing surveys.

We start with the motivation for our study in Sect. 2, discuss the coagulation model of planet formation and the moving snow line in Sect. 3, develop the disk evolution model in Sect. 4, and apply the model to super-Earth formation in Sect. 5. We end with a brief summary in Sect. 6 .

\section{Motivation: planet formation in the disk of a low-mass star}

To motivate our study, we contrast planet formation around low-mass stars and those moderately more massive that the sun (intermediate mass stars). As intermediate mass stars approach the main-sequence, the luminosity increases. Thus, the star is not at the main-sequence luminosity when protoplanets form in the first 0.1-1 Myr. The same is also true for low mass stars. For stars with masses $\lesssim 0.5 M_{\odot}$, the luminosity fades by a factor of 10-100 on the PMS track. During this evolution, the "snow line"- the point that separates the inner region of rocky planet formation from the outer region of icy planet formation-also moves inward.

Previous studies of planet formation (e.g. Ida and Lin 2005) have used the main-sequence luminosity when setting the location of the snow line. This assumption overlooks the movement of the snow line during the PMS contraction, and thus implies the snow line is too close to (far from) the central star for low-mass (intermediate-mass) stars.

Here we investigate the consequences of a moving snow line for low-mass stars, and how it sets where super-Earth building blocks form.

\section{Coagulation and the moving snow line}

In this section we briefly review the coagulation model of Solar System formation, where planets grow from repeated collisions and mergers of small objects in a circumstellar disk of gas and dust (Safronov 1969). Nearly all stars in sufficiently young clusters show these excesses (Haisch et al. 2000), which disappear over timescales of several million years as grains grow and the disk becomes optically thin.

To create a model disk in which planets grow, the Solar System planets are augmented to solar metallicity, and distributed evenly over concentric annuli. The radial profile of this "minimum mass solar nebula" (MMSN, Hayashi 1981) is

$\sigma(a)=\sigma_{0} a_{\mathrm{AU}}^{-3 / 2}$

where $\sigma_{0} \sim 8 \mathrm{~g} \mathrm{~cm}^{-2}$ is the surface density in solids at $1 \mathrm{AU}$, and $a_{\mathrm{AU}}$ is the radial distance from the star in units of AU. The surface density of gas follows a similar relation, increased by a factor of about 100 .

An important part of the MMSN model is the snow line at $\sim 3 \mathrm{AU}$. This distance marks where the temperature becomes low enough for ices to condense from the nebular gas, and where $\gtrsim M_{\oplus}$ protoplanets form in the Solar System.

Models of growth by coagulation commonly start with the MMSN. As the disk forms, micron-sized grains settle to the mid-plane of the disk. The settling rate depends on particle size, so grains begin to collide and stick as they settle, greatly increasing the rate of growth (Safronov 1969). Upon reaching the mid-plane, growth continues until 1$10 \mathrm{~km}$ "planetesimals" form (Weidenschilling 1980). The timescale for this growth is short-on the order of several thousand orbital periods-so repeated fragmentation is needed to explain the much longer observed disk lifetimes (Dullemond and Dominik 2005).

Once objects reach $\sim \mathrm{km}$ sizes, they are largely free from influence by the nebular gas (but see comments on migration below), and processes are dynamical.

The size distribution of objects evolves roughly as a power-law, with a few large objects, and many more small ones. Dynamical friction from small objects damps the orbital eccentricities of the largest objects, leading to "runaway growth", where the largest objects grow much faster relative to smaller objects (Wetherill and Stewart 1989). Throughout runaway growth, the largest protoplanets stir up the leftover planetesimals. Eventually, the leftovers have velocity dispersions comparable to the escape velocities of the largest protoplanets and runaway growth ends. The ensemble of planetesimals and protoplanets then enters "oligarchic" growth, where the largest objects-oligarchsaccrete at rates roughly independent of their size (Kokubo and Ida 1998).

During oligarchic growth, protoplanets become isolated from their surroundings. If an oligarch accretes all of the mass in an annulus with width $2 B R_{H}$, where the factor 
$B \sim 4$, and $R_{H}=a\left(M_{\mathrm{iso}} / 3 M_{\star}\right)^{1 / 3}$ is the Hill radius, its isolation mass is

$M_{\text {iso }} \approx 4 \pi a B R_{H} \sigma \propto(B \sigma)^{3 / 2} a^{3} M_{\star}^{-1 / 2}$,

(e.g. Lissauer 1993). However, both theoretical and numerical calculations show that oligarchic growth probably ends when oligarchs contain $\sim 50 \%$ of the total mass in solids, and dynamical friction from small objects no longer keeps the large objects in circular orbits (Goldreich et al. 2004; Kenyon and Bromley 2006).

To reach isolation, protoplanets must overcome type I migration, where the object is torqued by density waves excited in the gas disk (e.g. Tanaka et al. 2002). Type I migration timescales are typically very short-of the order $10^{4} \mathrm{yr}$ for the Jovian core. Icy protoplanets in general may suffer similar problems. However, the extremely short timescale has lead some researchers to include other effects. A likely important effect is magnetohydrodynamic (MHD) turbulence generated by the magnetorotational instability, a likely source of the viscosity needed to explain disk accretion onto young stars. MHD turbulence causes the migration of $\lesssim 10 M_{\oplus}$ protoplanets to become a random walk in semimajor axis rather than a steady decline, resulting in diffusion during oligarchic growth (see Papaloizou et al. 2007, and references therein for a recent review).

Growth beyond isolation is different in the inner and outer planetary regions of the Solar System. The boundary is marked by the snow line, where it becomes cold enough for ices to condense from the nebular gas into solids.

In the terrestrial region, isolated protoplanets have masses $\sim 0.1 M_{\oplus}$, and are rocky because volatile materials remain in the gas. In numerical models of the solar terrestrial zone, collisions and mergers of 10-20 oligarchs yield 2-5 planets with masses comparable to the mass of the Earth, on timescales of 10-100 Myr (e.g. Kenyon and Bromley 2006). The timescale for oligarchs to merge into planets is proportional to $P / \sigma$, where $P$ is the orbital period.

Outside the snow line, ice condensation enhances the surface density and promotes the formation of larger oligarchs. For a density $\rho \sim 1.5 \mathrm{~g} \mathrm{~cm}^{-3}$ and $\sigma \sim 3-6 \mathrm{~g} \mathrm{~cm}^{-2}$ at $5 \mathrm{AU}$, isolated oligarchs with $M_{\text {iso }} \sim 5-10 M_{\oplus}$ form on timescales $t_{\text {iso }} \sim 1$ Myr. These icy oligarchs accrete gas directly from the nebula and grow into gas giant planets in several million years (Pollack et al. 1996).

The time to reach isolation is important for two reasons. It determines when isolation occurs during the PMS contraction of the central star, and also determines whether a large protoplanet can form early enough to accrete a significant atmosphere. The timescale to reach isolation varies as (Goldreich et al. 2004)

$t_{\text {iso }} \propto \rho^{1 / 2} a^{3 / 2} \sigma^{-1 / 2}$.
Because the timescale for planetesimal and oligarch formation is short compared to the 0.1-1 Gyr PMS contraction time (e.g. Siess et al. 2000), the timing of planetesimal formation sets the nature of icy/rocky planets with distance from a star. Just outside the moving snow line, ice condensation increases $\sigma\left(M_{\text {iso }}\right)$ by a factor of $\sim 3(5)$ using solar oxygen abundances from Asplund et al. (2005). If the planetesimal density decreases by $2-3$ times, $t_{\text {iso }}$ decreases by a factor of $\sim 3$.

\section{Evolution of a disk around a contracting star}

In the standard MMSN model, $\sigma$ is fixed in time (e.g. Hayashi 1981). However, as a low-mass star contracts to the main-sequence, the snow line moves inward, and the surface density may change as the temperature drops below the ice condensation temperature.

To construct a model for disk evolution, we adopt

$\sigma(t)=\sigma_{0} \eta f_{\text {ice }} \frac{M_{\star}}{M_{\odot}} a_{\mathrm{AU}}^{-3 / 2}$

where $f_{\text {ice }}=3$ is the increase in surface density applied beyond the snow line, and $\eta$ allows us to vary the disk mass (relative to $M_{\star}$ ). For reference, with $\eta=1$ and $1 M_{\odot}$, the disk mass $M_{\text {disk }} \approx 0.025 M_{\star}$ (integrated to $60 \mathrm{AU}$ ). To derive the snow line distance, we adopt the temperature profile of a flat circumstellar disk, $T \propto T_{\star}\left(R_{\star} / a\right)^{3 / 4}$ (Kenyon and Hartmann 1987). Consistent with observations (e.g. Natta et al. 2000), we scale $\sigma$ and the disk mass linearly with the stellar mass. For further details, we refer the reader to Kennedy et al. (2006).

With these ingredients, we derive the evolution of $a_{\text {snow }}$, $\sigma, M_{\text {iso }}$, and $t_{\text {iso }}$ as the star contracts to the main sequence using Siess et al. (2000) PMS tracks. This evolution has two main features. Initially, the snow line is at $\sim 4 \mathrm{AU}$ from the luminous PMS star. Well inside the snow line, rocky oligarchs form and reach $M_{\text {iso }}$ before the star contracts significantly. At a few AU, the isolation timescale at the snow line is long compared to the initial contraction time. As the star contracts, ices condense out of the nebula and the snow line moves inward. For the inner region, this icy material coats the growing oligarchs, leftover planetesimals, and the surrounding debris with an icy veneer that may extend the oligarchic growth phase and produce more massive oligarchs. The snow line is at $\sim 1 \mathrm{AU}$ when the gas disk dissipates in 1$10 \mathrm{Myr}$. At a few $\mathrm{AU}$, ice condensation reduces the isolation timescale by increasing $\sigma$ and decreasing $\rho$.

\section{Super-Earth formation}

To explore the consequences of this picture, we consider a $0.25 M_{\odot} \mathrm{M}$ dwarf with a disk with mass $M_{\text {disk }} / M_{\star}=0.063$. 


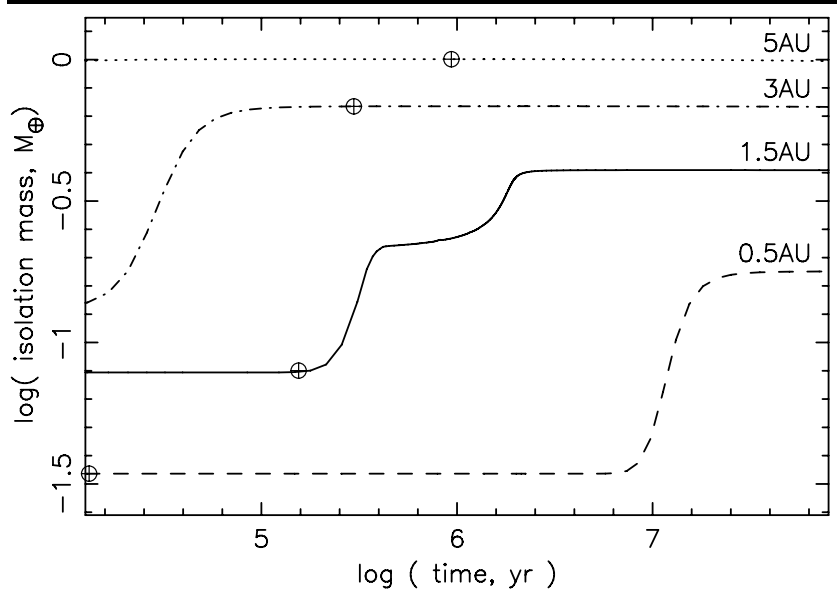

Fig. 1 Isolation masses at fixed radii over time around a $0.25 M_{\odot}$ star with $M_{\text {disk }} / M_{\star}=0.063$. The isolation time for each distance is marked by a $\oplus$. As the snow line moves inwards, ice condensation increases $\sigma$ (and hence $M_{\text {iso }}$ ), which leads to more rapid formation of more massive oligarchs. The dip in the $1.5 \mathrm{AU}$ line at $\sim 1 \mathrm{Myr}$ is caused by deuterium burning in the star briefly stabilising the contraction

This disk is $\eta=2.5$ times more massive than the $M_{\star}$ scaled MMSN, and about half the mass of the most massive observed disks (e.g. Natta et al. 2000). Alternatively, the disk could have an increased metallicity, and retain $M_{\text {disk }}=$ $0.025 M_{\star}$. Figure 1 shows isolation mass evolution for this system at several distances from the central star. The figure shows clear increases when the snow line crosses specific points in space and ices condense out of the gas.

Interior to $a_{\text {snow }}$ at isolation ( $a \lesssim 1-2 \mathrm{AU}$ ), rocky oligarchs with $M_{\text {iso }} \sim 0.1 M_{\oplus}$ form in $\lesssim 10^{5}$ yr. This time is probably somewhat limited by the disk and planetesimal formation time, and not as short as (3) and the figure suggest. At 1.5 AU, continued movement of the snow line replenishes $\sigma$ and enables further growth of oligarchs to $M_{\text {iso }} \sim 0.3 M_{\oplus}$ in several $10^{5}$ yr. At $a \sim 2-3 \mathrm{AU}$, ice condensation during runaway growth promotes the formation of oligarchs with $M_{\text {iso }} \sim 0.5 M_{\oplus}$ in $\sim 10^{5} \mathrm{yr}$.

This analytic prescription for protoplanet growth suggests that oligarchs with $M_{\text {iso }} \sim 0.1-0.5 M_{\oplus}$ can form at $\sim 1-3$ AU in $\lesssim 1$ Myr. The model predicts $\sim 10$ oligarchs at 1.5-4 AU. Thus, the building blocks for observable superEarths can form on timescales much shorter than disk lifetimes derived from measurements of dust emission from low-mass PMS stars (e.g. Plavchan et al. 2005). We find it unlikely that icy protoplanets form interior to $\sim 1 \mathrm{AU}$ for $0.25 M_{\odot}$ stars, in contrast to Ida and Lin (2005).

There are two main considerations for the final stages of coalescence into super-Earths. Goldreich et al. (2004) point out that interactions are more likely to lead to ejections when the escape velocity from a planet is greater than the local orbital velocity, which allows us to set planetary mass limits as a function of distance from the star. In this picture we expect to form $\sim 5 M_{\oplus}$ planets at $1-2 \mathrm{AU}, \sim 3 M_{\oplus}$ planets at
2-3 AU, and 1-2M $M_{\oplus}$ planets at 3-4 AU. If we simply adapt the collisional history of Earth formation to a planetesimal disk around a $0.25 M_{\odot}$ star, mergers of $\sim 10$ oligarchs should yield planets with masses $\sim 1-2 M_{\oplus}$ at $1 \mathrm{AU}$ and $\sim 3-5 M_{\oplus}$ at $2.5 \mathrm{AU}$.

To consider whether oligarchs can merge into superEarths on reasonable timescales, we use the scaling relation for Earth formation $t_{\text {form }} \propto 10-100 P / \sigma_{0}$ Myr. The expected merger timescale for oligarchs at $1-3 \mathrm{AU}$ around a $0.25 M_{\odot}$ star is $\sim 2-5$ times longer than for the terrestrial zone around a solar-type star. Thus, coagulation can produce super-Earths around low-mass stars on timescales of $\sim 50-500 \mathrm{Myr}$.

The main uncertainties in our picture are the probability of the initial disk mass and the details of the final accretion stage when $1-2 M_{\oplus}$ planets evolve into $3-5 M_{\oplus}$ planets. Since we expect planet masses to increase with disk mass, we naturally expect to discover planets originating from relatively massive disks first. Observations of larger samples can yield better estimates for the range of initial disk masses for low-mass stars and for the $M_{\text {disk }}-M_{\star}$ relation. Detailed numerical simulations can provide better estimates of the masses and formation timescales for super-Earths.

Observations of comets may argue for a smaller (a factor 2) jump in surface density at the snow line (Küppers et al. 2005), with an increase in $M_{\text {iso }}$ of $\sim 3$. While the difference in (proto)planet masses across the snow line will likely be less dramatic, super Earth-mass planets will still form beyond it.

In this picture we have ignored migration, which may be important in the first $\sim 1-10 \mathrm{Myr}$ while the gas disk is still present. Type I theory (e.g. Tanaka et al. 2002), predicts a single $\sim 0.5 M_{\oplus}$ protoplanet will migrate into the central star in a few $10^{5} \mathrm{yr}$ in the absence of MHD turbulence, similar to its formation time. In the increased metallicity scenario for $\eta$, the migration timescale becomes a few times longer than the isolation timescale. However, effects such as MHD turbulence will likely reduce migration of these low mass protoplanets to a random walk, allowing them to survive until the gas disk is dissipated and migration stops.

\section{Summary}

We have developed an analytic prescription for snow line evolution, and planet formation by coagulation around lowmass stars, and applied it to super-Earth formation around a $0.25 M_{\odot}$ star. At $1-5 \mathrm{AU}$, isolated oligarchs can grow to masses $\sim 0.1-1 M_{\oplus}$ in $\sim 0.1-1 \mathrm{Myr}$, short compared to the $\sim 100$ Myr PMS contraction time. These oligarchs merge into super-Earths with masses $\sim 2-5 M_{\oplus}$ as the star contracts to the main sequence. Thus, coagulation can produce planetary systems similar to those detected in recent microlensing events. 
Acknowledgements We acknowledge support from the NASA Astrophysics Theory Program through grant NAG5-13278 (SK, BB) and an Australian Postgraduate Award (GK). We thank T. Currie, M. Geller, the ANU Planetary Science Institute planet group for helpful comments on the project. We thank the referee Pawel Artymowicz for a thorough review of the manuscript.

\section{References}

Asplund, M., Grevesse, N., Sauval, A.J.: The solar chemical composition. In: Barnes, T.G. III, Bash, F.N. (eds.) Cosmic Abundances as Records of Stellar Evolution and Nucleosynthesis. Astronomical Society of the Pacific Conference Series, vol. 336, p. 25 (2005)

Beaulieu, J.P., et al.: Discovery of a cool planet of 5.5 Earth masses through gravitational microlensing. Nature 439, 437-440 (2006). doi:10.1038/nature04441

Boss, A.P.: Rapid formation of super-Earths around $\mathrm{M}$ dwarf stars. Astrophys. J. 644, L79-L82 (2006). doi:10.1086/505533

Dullemond, C.P., Dominik, C.: Dust coagulation in protoplanetary disks: a rapid depletion of small grains. Astron. Astrophys. 434, 971-986 (2005). doi:10.1051/0004-6361:20042080

Goldreich, P., Lithwick, Y., Sari, R.: Final stages of planet formation. Astrophys. J. 614, 497-507 (2004). doi:10.1086/423612

Gould, A., et al.: Microlens OGLE-2005-BLG-169 implies that cool Neptune-like planets are common. Astrophys. J. 644, L37-L40 (2006). doi:10.1086/505421

Haisch, K.E. Jr., Lada, E.A., Lada, C.J.: A near-infrared L-band survey of the young embedded cluster NGC 2024. Astron. J. 120, 13961409 (2000). doi:10.1086/301521

Hayashi, C.: Structure of the solar nebula, growth and decay of magnetic fields and effects of magnetic and turbulent viscosities on the nebula. Prog. Theor. Phys. Suppl. 70, 35-53 (1981)

Ida, S., Lin, D.N.C.: Toward a deterministic model of planetary formation. III. Mass distribution of short-period planets around stars of various masses. Astrophys. J. 626, 1045-1060 (2005). doi:10.1086/429953

Kennedy, G.M., Kenyon, S.J., Bromley, B.C.: Planet formation around low-mass stars: the moving snow line and super-Earths. Astrophys. J. 650, L139-L142 (2006). doi:10.1086/508882

Kenyon, S.J., Bromley, B.C.: Terrestrial planet formation. I. The transition from oligarchic growth to chaotic growth. Astron. J. 131, 1837-1850 (2006). doi:10.1086/499807
Kenyon, S.J., Hartmann, L.: Spectral energy distributions of T Tauri stars-disk flaring and limits on accretion. Astrophys. J. 323, 714-733 (1987). doi: $10.1086 / 165866$

Kokubo, E., Ida, S.: Oligarchic growth of protoplanets. Icarus 131, 171-178 (1998). doi:10.1006/icar.1997.5840

Küppers, M., et al.: A large dust/ice ratio in the nucleus of comet 9P/Tempel 1. Nature 437, 987-990 (2005). doi:10.1038/nature 04236

Laughlin, G., Bodenheimer, P., Adams, F.C.: The core accretion model predicts few jovian-mass planets orbiting red dwarfs. Astrophys. J. 612, L73-L76 (2004). doi:10.1086/424384

Lissauer, J.J.: Planet formation. Annu. Rev. Astron. Astrophys. 31, 129-174 (1993). doi:10.1146/annurev.aa.31.090193.001021

Natta, A., Grinin, V., Mannings, V.: Properties and evolution of disks around pre-main-sequence stars of intermediate mass. In: Protostars and Planets IV, p. 559 (2000)

Papaloizou, J.C.B., Nelson, R.P., Kley, W., Masset, F.S., Artymowicz, P.: Disk-planet interactions during planet formation. In: Reipurth, B., Jewitt, D., Keil, K. (eds.) Protostars and Planets V, pp. 655668 (2007)

Plavchan, P., Jura, M., Lipscy, S.J.: Where are the M dwarf disks older than 10 million years? Astrophys. J. 631, 1161-1169 (2005). doi: $10.1086 / 432568$

Pollack, J.B., Hubickyj, O., Bodenheimer, P., Lissauer, J.J., Podolak, M., Greenzweig, Y.: Formation of the giant planets by concurrent accretion of solids and gas. Icarus 124, 62-85 (1996). doi:10.1006/icar.1996.0190

Safronov, V.S.: Evoliutsiia doplanetnogo oblaka (1969)

Siess, L., Dufour, E., Forestini, M.: An internet server for pre-main sequence tracks of low- and intermediate-mass stars. Astron. Astrophys. 358, 593-599 (2000)

Tanaka, H., Takeuchi, T., Ward, W.R.: Three-dimensional interaction between a planet and an isothermal gaseous disk. I. Corotation and lindblad torques and planet migration. Astrophys. J. 565, 12571274 (2002). doi:10.1086/324713

Weidenschilling, S.J.: Dust to planetesimals—settling and coagulation in the solar nebula. Icarus 44, 172-189 (1980). doi:10.1016/0019-1035(80)90064-0

Wetherill, G.W., Stewart, G.R.: Accumulation of a swarm of small planetesimals. Icarus 77, 330-357 (1989). doi:10.1016/ 0019-1035(89)90093-6 